\title{
PVSRIPO, A Potential New Tool in Our Fight against GBM
}

\author{
Jack Korleski ${ }^{1,2}$, Hernando Lopez-Bertoni ${ }^{1,2 *}$ \\ 1. Hugo W. Moser Research Institute at Kennedy Krieger \\ 2. Department of Neurology, Johns Hopkins University School of Medicine.
}

Corresponding author: Hernando Lopez-Bertoni, Email: Lopezbertoni@kennedykrieger.org

GBM is the most common primary malignant brain cancer. Standard of care therapy includes maximum total resection with adjuvant radiation and chemotherapy with temozolomide ${ }^{[1]}$. GBM inevitably recurs and options to treat recurrent GBM are limited to resection of bulk tumor surgeries, radiation, and experimental targeted therapies. Despite these treatment options the median survival remains poor at 15 months $\mathrm{s}^{[2]}$. Given the poor prognosis, new therapies are needed to expand the arsenal of treatment options for GBM.

One area of ongoing interest for cancer management is the use of oncolytic viruses as therapeutic modalities ${ }^{[3]}$. This class of viruses specifically target neoplastic cells and can eliminate them by different mechanisms, including induction of cell death via direct cellular toxicity, induction of tumor autoimmunity, or can be engineered to deliver high doses of chemotherapy ${ }^{[3,4]}$. In the context of GBM, a number of viruses are being studied for therapeutic development. Many of these include viruses known to target the CNS, such a poliovirus, HSV, measles and adenovirus ${ }^{[3,4]}$. Of these, PVSRIPO, a modified poliovirus has shown clinical potential in recent clinical trials ${ }^{[5,6]}$.

Poliovirus is a positive sense, single stranded RNA virus that is transmitted via the fecal-oral route. The virus is well known to cause CNS infection with many patients experiencing significant paralysis leading to respiratory failure ${ }^{[7]}$. The development of poliovirus for the treatment of GBM was sparked by 2 very attractive molecular pathophysiology characteristics of infection: (i) the virus binds CD155 for neuronal entry and (ii) it binds host ribosomes with an internal ribosomal entry site (IRES) at the 5 ' $\mathrm{UTR}^{[5,6,8]}$. These features are important because both glioma cells and antigen presenting cells express high levels of CD155 ${ }^{[9]}$ and early experiments showed that mutating the IRES leads to decreased neuronal toxicity ${ }^{[8]}$. These observations gave rise to the

(D) (C) The Author(s) 2022. Open Access This article is licensed under a Creative Commons Attribution 4.0 International License (https://creativecommons.org/licenses/by/4.0/), which permits unrestricted use, sharing, adaptation, distribution and reproduction in any medium or format, for any purpose, even commercially, as long as you give appropriate credit to the original author(s) and the source, provide a link to the Creative Commons license, and indicate if changes were made. 
hypothesis that PVSRIPO could be a novel therapeutic for GBM. To test this hypothesis, the PVSRIPO virus was engineered by replacing the poliovirus IRES with that of rhinovirus, which reduced neurotoxicity ${ }^{[10]}$. Treating neoplastic cells with this recombinant PVSRIPO showed increased viral growth and mice treated intratumorally had increased survival in an orthotopic model of $\mathrm{GBM}^{[10,11]}$. Subsequent studies in melanoma cell models with CD155 overexpression showed both dendritic cell activation with a type I IFN pro-inflammatory response and development of tumor specific effector T-cells in response to treatment with PVSRIPO ${ }^{[12]}$. These data suggest a three-fold mechanism of action for PVSRIPO: (i) the virus can target glioma cells, replicate, and induce cellular cytotoxicity; (ii) the virus can active antigen presenting cells and induce a local pro-inflammatory response within the tumor microenvironment (iii) the virus can induce an adaptive immune response that leads to effector $\mathrm{T}$ cells that target tumor antigens, ${ }^{[12]}$.

These promising results pushed this pre-clinical therapeutic to a phase $1 / 2$ clinical trial to test if intra-tumoral delivery of PVSRIPO had a clinical benefit in recurrent GBM patients6. This trial showed that compared to historical controls, the median overall survival of patients that received PVSRIPO was non-significant. However, there was a significant increase in the number of long-term survivors ( $>36$ months) in patients treated with PVSRIPO, with $21 \%$ of patients treated with PVSRIPO surviving at 60 months compared to none of the historical controls ${ }^{[6]}$. Further analysis of patients treated with PVSRIPO also showed an increased rate of responsiveness to a re-challenge with chemotherapy ${ }^{[6]}$. While not fully understood, this could indicate either selective targeting of a subpopulation of glioma cells that drive chemotherapy resistance or that PVSRIPO has better replicative ability in certain subtypes of GBM. Additionally, given the pre-clinical studies showing local pro-inflammatory and effector $\mathrm{T}$ cell responses from PVSRIPO ${ }^{[12]}$, concomitant treatment of immunomodulatory therapies such as PD-1 immune checkpoint inhibitors are predicted to increase the responsiveness to therapy. These very exciting results prompted ongoing clinical trials for survival benefit of PVSRIPO treatment alone and in combination with chemotherapy or PD-1 inhibitors ${ }^{[2]}$.

The collective data for PVSRIPO in treating GBM show both positive future directions and limitations. The challenges facing PVSRIPO are similar to many other therapies in treating GBM, the original pre-clinical studies with PVSRIPO showed poor efficacy when PVSRIPO was delivered systemically ${ }^{[10]}$. While intra-tumoral delivery allows for increase local concentration of virus, a variety of mechanisms limit the ability for the virus to spread to the leading edges of the tumor, such as diffusion limitations ${ }^{[13]}$. Additionally, as seen with recent trial for HSV treatment in pediatric high-grade glioma, presence of neutralizing antibodies to the virus limited clinical responsiveness to therapy ${ }^{[14]}$. Given mass vaccinations against poliovirus, the widespread presence of neutralizing antibodies to poliovirus may be a significant limitation in the ability to expand therapeutic responses to more than the $21 \%$ seen in clinical trials. However, the fact that there was a therapeutic response despite widespread vaccination gives weight to the preclinical model of viral infection leading to antigen presenting cell activation and subsequent tumor neo-antigen specific $\mathrm{T}$ cell responses. Understanding the neoantigen specific T-cells present in the patients who did respond to treatment could not only give a better understanding of how to expand the therapeutic potential of PSVRIPO, but could also help develop novel PVSRIPO-independent immunotherapies, such as CAR T-cell therapy.

\section{REFERENCES:}

[1] Ostrom, Q. T. et al. CBTRUS Statistical Report: Primary Brain and Other Central Nervous System Tumors Diagnosed in the United States in 2013-2017. Neuro Oncol 22, iv1-iv96, doi:10.1093/neuonc/noaa200 (2020).

[2] Cruz Da Silva, E., Mercier, M. C., Etienne-Selloum, N., Dontenwill, M. \& Choulier, L. A Systematic Review of Glioblastoma-Targeted Therapies in Phases II, III, IV Clinical Trials. Cancers (Basel) 13, doi:10.3390/ cancers 13081795 (2021). 
[3] Haseley, A., Alvarez-Breckenridge, C., Chaudhury, A. R. \& Kaur, B. Advances in oncolytic virus therapy for glioma. Recent Pat CNS Drug Discov 4, 1-13, doi:10.2174/157488909787002573 (2009).

[4] Martikainen, M. \& Essand, M. Virus-Based Immunotherapy of Glioblastoma. Cancers (Basel) 11, doi:10.3390/cancers11020186 (2019).

[5] Carpenter, A. B., Carpenter, A. M., Aiken, R. \& Hanft, S. Oncolytic virus in gliomas: a review of human clinical investigations. Ann Oncol, doi:10.1016/j.annonc.2021.03.197 (2021).

[6] Desjardins, A. et al. Recurrent Glioblastoma Treated with Recombinant Poliovirus. N Engl J Med 379, 150161, doi:10.1056/NEJMoa1716435 (2018).

[7] De Jesus, N. H. Epidemics to eradication: the modern history of poliomyelitis. Virol J 4, 70, doi:10.1186/1743-422X-4-70 (2007).

[8] Gromeier, M., Alexander, L. \& Wimmer, E. Internal ribosomal entry site substitution eliminates neurovirulence in intergeneric poliovirus recombinants. Proc Natl Acad Sci U S A 93, 2370-2375, doi:10.1073/pnas.93.6.2370 (1996).

[9] Chandramohan, V. et al. Validation of an Immunohistochemistry Assay for Detection of CD155, the Poliovirus Receptor, in Malignant Gliomas. Arch Pathol Lab Med 141, 1697-1704, doi:10.5858/arpa.20160580-OA (2017).

[10] Gromeier, M., Lachmann, S., Rosenfeld, M. R., Gutin, P. H. \& Wimmer, E. Intergeneric poliovirus recombinants for the treatment of malignant glioma. Proc Natl Acad Sci U S A 97, 6803-6808, doi:10.1073/ pnas.97.12.6803 (2000).

[11] Dobrikova, E. Y. et al. Recombinant oncolytic poliovirus eliminates glioma in vivo without genetic adaptation to a pathogenic phenotype. Mol Ther 16, 1865-1872, doi:10.1038/mt.2008.184 (2008).

[12] Brown, M. C. et al. Cancer immunotherapy with recombinant poliovirus induces IFN-dominant activation of dendritic cells and tumor antigen-specific CTLs. Sci Transl Med 9, doi:10.1126/scitranslmed.aan4220 (2017).

[13] Haumann, R., Videira, J. C., Kaspers, G. J. L., van Vuurden, D. G. \& Hulleman, E. Overview of Current Drug Delivery Methods Across the Blood-Brain Barrier for the Treatment of Primary Brain Tumors. CNS Drugs 34, 1121-1131, doi:10.1007/s40263-020-00766-w (2020).

[14] Friedman, G. K. et al. Oncolytic HSV-1 G207 Immunovirotherapy for Pediatric High-Grade Gliomas. N Engl J Med 384, 1613-1622, doi:10.1056/NEJMoa2024947 (2021). 